\title{
Characteristics of Shield Materials for Wireless Power Transfer
}

\author{
In Chang Chu ${ }^{1}$ and Jinseong Jeong ${ }^{2 *}$ \\ ${ }^{1}$ Global EMI/EMC Team, 3M Korea Innovation Center, Hwaseong-Si 445-179, Korea \\ ${ }^{2}$ School of Electrical and Computer Engineering, University of Seoul, Seoul 130-743, Korea
}

(Received 23 March 2014, Received in final form 11 June 2014, Accepted 12 June 2014)

\begin{abstract}
In this paper, we examine the electrical and magnetic properties of three different types of shield materials used for wireless power transfer systems: namely, FeSiAl-composite, NiZn-ferrite, and FeSi-amorphous types. The power transfer efficiency and resistance of an RX coil are measured, while varying the shield thickness. For all three types, a thicker shield provides better power transfer efficiency. Analysis of the measurements shows that the FeSiAl-composite type is suitable for systems with size limitation. In terms of magnetic properties, the FeSiamorphous type shows the best features, and is suited to high power applications. This work can be used as a guideline to select suitable shielding material in various wireless power transfer systems.
\end{abstract}

Keywords : wireless power transfer system, electromagnetic interference prevention, shield material

\section{Introduction}

Wireless power transfer (WPT), also called contactless electrical energy transmission (CEET), is a technique to deliver electrical power or energy from a source to a load without any wired connection. WPT is advantageous in many applications where wires should not be used. For example, a WPT system is employed to eliminate plug-in power connections in wet or hazardous environments, to avoid such risks as electrical shock, short-circuiting, or sparking [1]. Also, WPT can be applied to biological devices, such as artificial hearts and endoscopic capsules $[2,3]$. In three-dimensional (3D) TVs, shutter glasses using the active-switching method also benefit from wireless charging [4]. There are many more places where the WPT can be conveniently deployed, including electric vehicle charging, and the wire-free operation of home appliances.

The implementation of a WPT system is based on the fundamental principle of electromagnetics, and can be classified according to the electromagnetic coupling methods. Among them, the inductive coupling method is used in a wide range of applications, including electric vehicle and home appliances. Despiteits high efficiency $[5,6]$, in most cases the transmission distance is limited

(C)The Korean Magnetics Society. All rights reserved.

*Corresponding author: Tel: +82-2-6490-2347

Fax: +82-2-6490-2314, e-mail: jsjeong@uos.ac.kr to less than a centimeter. On the other hand, the resonant coupling method suggested by Kurs et al. achieves both high efficiency and longer transmission distance [7]. However, the size of coils required for magnetic resonance between two or more objects is often too great.

In addition to the efficiency and the transmission distance, it is crucial to satisfy various regulations on safety levels and electromagnetic interference (EMI). In implementing an efficient and safe WPT system, the shield material play an important role, since it reduces EMI between electronic circuits inside or outside the system, and it also prevents electromagnetic power leakage, while maximizing power transfer efficiency. Therefore, it is very important to fully understand the characteristics of shield materials, in order to apply suitable materials for the various WPT systems.

In this study, we investigate the electrical and magnetic properties of three different types of shield materials used for inductively coupled WPT systems. In addition to property characterization, we also observe their microscopic structures by cross-sectional scanning electron microscope (SEM), and discuss the relationship between their structures and characteristics. The outcome of this study can be used as a guideline to select a suitable shield material, depending on the environment where the WPT system is employed.

\section{Experimental Methods}

In this experiment, power is transferred via magnetic 


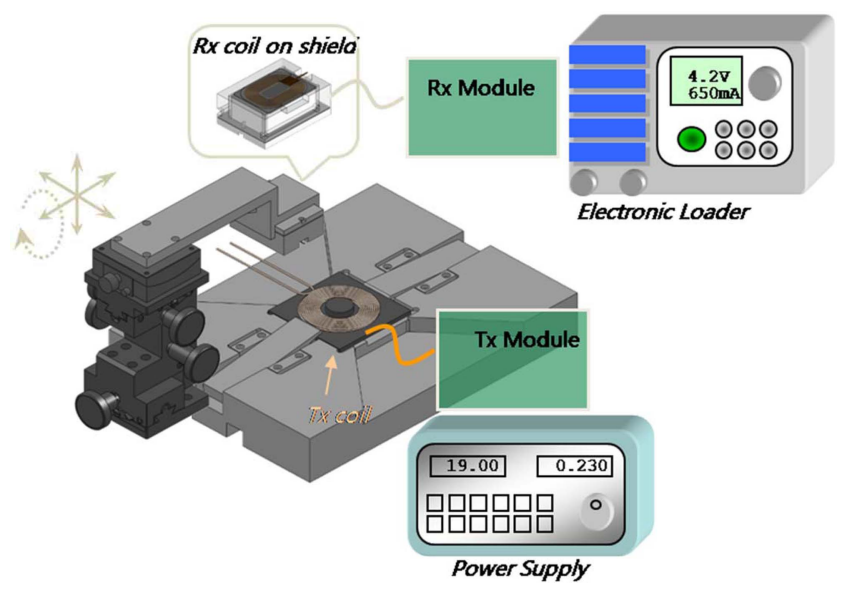

Fig. 1. (Color online) WPT test bed setup for power transfer efficiency and RX coil resistance.

induction between two air-core coils. The electrical and magnetic characteristics of three types of shield materials, FeSiAl-composite, NiZn-ferrite, and FeSi-amorphous are compared. The experimental system is composed of two coils, one in the transmitter (TX), and the other in the receiver (RX), with a power supply, and an electronic load. Figure 1 shows the system staged on our homemade test bed. The TX coil on the bottom plate is connected to a power supply, while the RX coil in the floating stage is connected to the load. The shield material under test is in contact with the side of the RX coil, but is relatively far from the TX coil. The position of the RX coil can be optimized, using a three-axis translational stage. In this configuration, the experiment is performed in a consistent and systematic manner. The efficiency of TX-to-RX power transfer, as well as the resistance of the RX coil, is measured for each shield material, while changing the thickness of shielding plates. The size of the TX and RX coils is selected according to the specifications of the wireless power consortium. For the power transmission, an input voltage of $12 \mathrm{~V}$ is applied to the TX coil, and the operating frequency is varied in the range of $100-200 \mathrm{kHz}$.

\section{Results and Discussion}

In this study, a FeSiAl-composite type, a NiZn-ferrite type, and a FeSi-amorphous type are used for shield materials, to investigate how those materials affect the electrical properties of the WPT system, by measuring the power transfer efficiency and resistance of the RX coil.

Figure 2 shows the power transfer efficiency as a function of the thickness of each shield material. As shown in the figure, the FeSiAl-composite type shows higher efficiency than the other two materials for a given thickness.

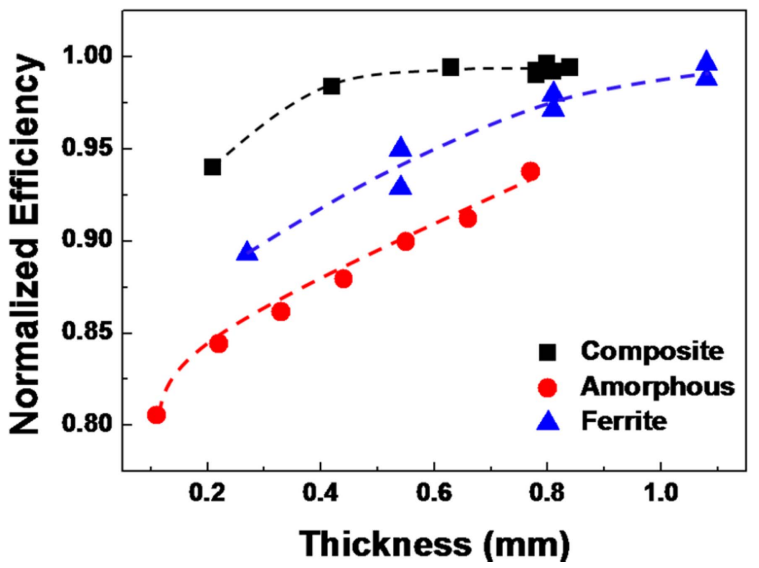

Fig. 2. (Color online) Power transfer efficiency as a function of shielding thickness (the dotted lines are generated by curve fitting).

The efficiency improves as the shield becomes thicker for all three cases, which is manifested by the dotted lines generated by curve fitting. Although this experiment tells us that a thicker shield is desirable to increase the power transfer efficiency, in many cases the thickness is restricted by dimensional limitations imposed on electronic products.

The relationship between the power transfer efficiency and the resistance of the RX coil for each shield material is depicted in Fig. 3, where a cluster of the FeSiAlcomposite type shows high efficiency and low resistance. The FeSiAl-composite type reduces the resistance of the RX coil lower than the other types, which, in turn, contributes to the efficiency enhancement. The NiZn-ferrite type shows better performance than the FeSi-amorphous type, in terms of reducing the resistance of the RF coil. However, the efficiency spreads from $85 \%$ to $100 \%$, which means a significant amount of electromagnetic power can

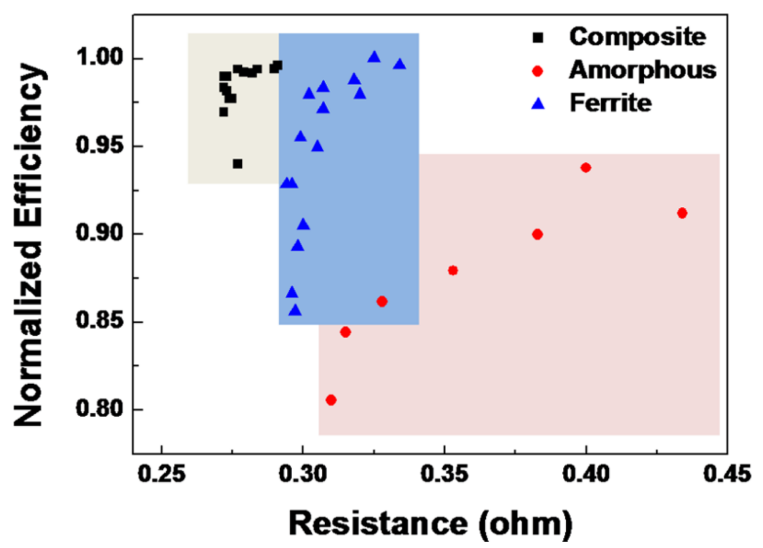

Fig. 3. (Color online) Power transfer efficiency as a function of RX coil resistance (the clusters are shaded according to each type). 


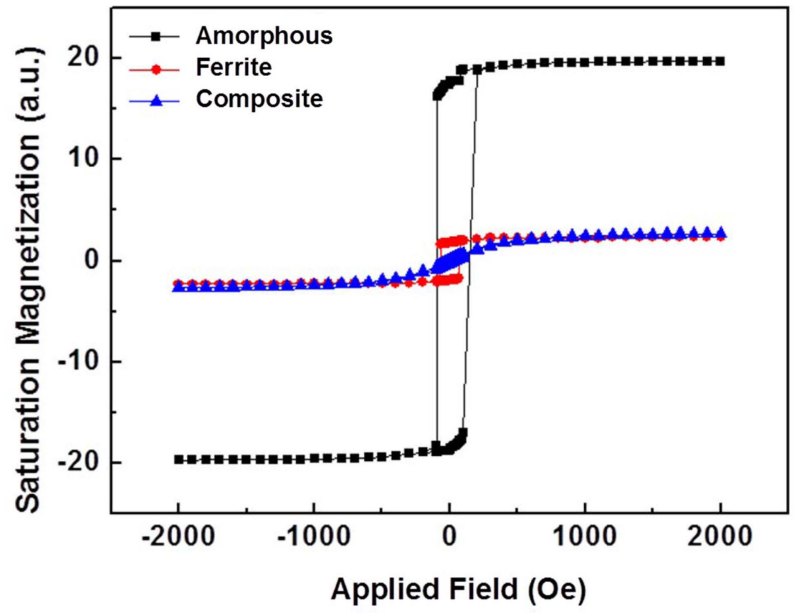

Fig. 4. (Color online) $\mathrm{M}-\mathrm{H}$ curves of the magnetic materials used in shields.

leak, depending on the shielding thickness. This experiment shows that the proper selection of a shield material is very important to maximize the power transfer efficiency in an induction-based WPT system. The FeSiAlcomposite gives higher efficiency, regardless of its thickness. Therefore, the FeSiAl-composite is suitable for systems with device-size limitation.

In addition, the magnetic properties of the three shield materials are measured, since the characteristics of the WPT system can be affected by the magnetic properties, such as permeability and magnetic flux density, which may change the formation of a closed magnetic circuit. A typical M-H curve is shown in Fig. 4, where the saturation magnetization $\left(M_{s}\right)$ of the FeSi-amorphous type is 10 times larger than that of the other two materials, while the $M_{s}$ of the FeSiAl-composite type is slightly larger than that of the NiZn-ferrite type, by $5 \%$. However, the coercivity $\left(H_{c}\right)$ value barely changed as a function of shield material. The $H_{c}$ values of FeSi-amorphous, NiZn-ferrite, and $\mathrm{FeSiAl}$-composite type were 2, 2, and $4 \mathrm{Oe}$, respectively. According to this $\mathrm{M}-\mathrm{H}$ curve, the FeSi-amorphous type will be able to respond to a higher magnetic field, compared with the other materials. Therefore, the FeSiamorphous is suitable for high power WPT applications.

Figure 5 shows the microscopic structures of shield materials by SEM. The FeSiAl-composite type, as shown in Fig. 5(a), is composed of an aggregation of rod-shaped flakes, which determine the permeability. The shape and size of flakes are very important in controlling the properties of composite materials. Normally, in the composite type, long flakes represent high permeability, while short flakes represent low permeability. Therefore, to obtain high permeability in the composite type, the shape aniso-
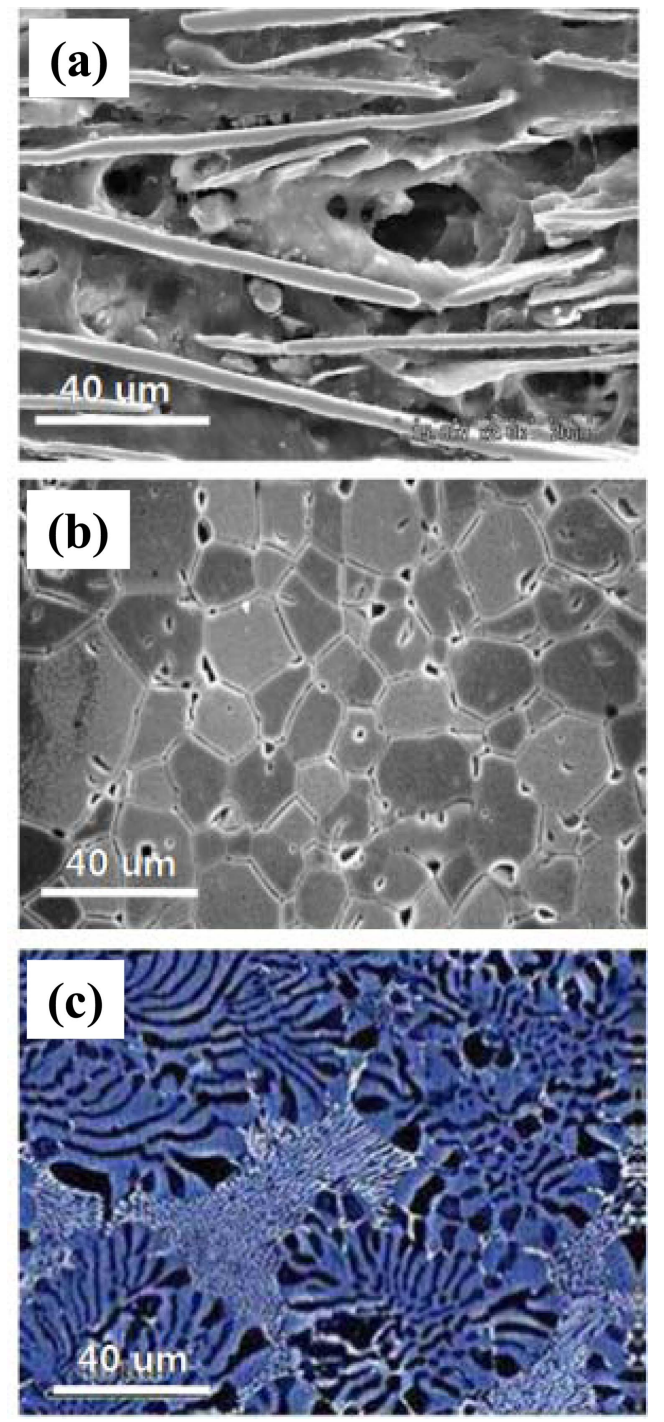

Fig. 5. (Color online) Cross-sectional scanning electron microscope (SEM) images of shield materials. (a) FeSiAl-composite, (b) NiZn-ferrite, and (c) FeSi-amorphous type.

tropy of the flakes needs to be large. Fig. 5(b) shows the grains and the grain boundaries of the NiZn-ferrite type. The grain size affects the permeability and resistivity in ferrite materials. Alarge grain contributes to high permeability, and a narrow grain boundary offers low resistivity. These sizes can be controlled by the annealing rate, cooling rate, etc. Therefore, in order to improve the properties of composite materials, optimization of the heat treatment process is indispensable. Finally, as shown in Fig. 5(c), the FeSi-amorphous type doesn't have crystallographic directions. Therefore, in the amorphous type, proper selection of the base material is important, to control the properties of the shield.

From a practical point of view, various factors must be considered when selecting a suitable shield material. The 
FeSiAl-composite type shield is flexible, and gives good permittivity, but it has the disadvantage of poor permeability compared with the other shields, because extra ingredients should be mixed to make the composite type shield. The NiZn-ferrite type shield has the advantage of high permeability. However, it is too brittle to be used in those cases that are vulnerable to mechanical impact or shock. The FeSi-amorphous type shield has high permeability, but it gives high current loss, caused by the eddy current due to low resistivity. In WPT, therefore, it is very important to select suitable materials, depending on key environmental issues, such as product thickness, shock vulnerability, frequency, power, acting temperature, and neighboring equipment.

\section{Conclusions}

In this paper, we examined how the electric and magnetic properties of shield materials affect a WPT system, and investigated the magnetic properties and microscopic structures of shield materials. We tested three materials typically used for WPT: composite, ferrite, and amorphous types. For all three types, a thicker shield provides better power transfer efficiency. The FeSiAl-composite type is suitable for systems with size limitation. In terms of magnetic properties, the FeSi-amorphous type shows the best features, and is suitable for high power applications. Our experimental results can be used as a guideline to select suitable shielding material, according to the environment where the WPT system is employed.

\section{Acknowledgments}

This work was supported by the 2011 Research Fund of the University of Seoul.

\section{References}

[1] D. A. G. Pedder, A. D. Brown, and J. A. Skinner, IEEE Trans. Ind. Electron. 46, 23 (1999).

[2] Y. Jang and M. M. Jovanovic, IEEE Trans. Ind. Electron. 50, 520 (2003).

[3] Y. P. Su, X. Liu, and S. Y. R. Hui, IEEE Trans. Power Electron. 24, 1115 (2009).

[4] H. J. Choi, 3D Research 2, 02004 (2011).

[5] Z. N. Low, R. A. Chinga, R. Tseng, and J. Lin, IEEE Trans. Ind. Electron. 56, 1801 (2009).

[6] J. Sallan, J. L. Villa, A. Llombart, and J. F. Sanz, IEEE Trans. Ind. Electron. 56, 3060 (2009).

[7] A. Kurs, A. Karalis, R. Moffatt, J. D. Joannopolos, P. Fisher, and M. Soljacic, Science 317, 83 (2007). 